\title{
Experimental study of the gap between track slab and cement asphalt mortar layer in CRTS I slab track
}

\author{
Tao Wang ${ }^{1} \cdot$ Hengqiong $\mathrm{Jia}^{1} \cdot \mathrm{Zike}^{\mathrm{Liu}}{ }^{1} \cdot \mathrm{Zhao} \mathrm{Wei}^{1} \cdot \mathrm{Xiao}^{\mathrm{Xie}}{ }^{1} \cdot$ Shaoliang $\mathrm{Wu}^{1} \cdot$ \\ Haiyan $\mathrm{Li}^{1}$
}

Received: 16 January 2017/Revised: 1 June 2018/Accepted: 3 June 2018/Published online: 25 June 2018

(C) The Author(s) 2018

\begin{abstract}
Gap exists in the interface of cement asphalt emulsion mortar and CRTS I track slab universally, which is more severe at four corners than other parts of the track slab. In this work, the temperature and elevation of CRTS I slab track with and without rail were measured continuously to study the influence mechanism of rail on the gap. The results show that the alternating temperature gradient of track slab is the main reason that causes the gap, and laying rail can efficiently decrease the gap size in the slab track without rail. Compared with the slab track without rail, the maximum elevation occurred at the corner, the maximum gap width and the maximum gap depth of the slab track with rail laid were decreased by $0.45 \mathrm{~mm}(25.7 \%), 0.75 \mathrm{~mm}(46.6 \%)$ and $9.5 \mathrm{~mm}(59.4 \%)$, respectively; meanwhile, the disqualification ratio at corners was reduced to $5.9 \%$, which is $50 \%$ less than that of the track without rail. When elevation mismatch occurs in adjacent track slabs, a gasket should be placed at rail-bearing bed below the track slab in order to avoid the lower slab being dragged up by the higher slab and the further occurrence of new gap.
\end{abstract}

Keywords Ballastless track · Rail · Track slab · CA mortar - Gap

China railway track system I type (CRTS I) ballastless track is one of the main structures used in high-speed railways owing to its simple structure, convenient construction and easy maintenance; it also has the longest

Tao Wang

027wangtao@163.com

1 Metals and Chemistry Research Institute, Academy of Railway Sciences China, Beijing 100081, China application mileage in the world [1-4]. However, the gap tends to emerge in the interface between cement asphalt (CA) mortar and slab due to the shrinkage of CA mortar and ambient temperature variation, which is more severe at the four corners of the track slab [5-8].

Some theoretical research on track structures shows that the gap, which originates from periodic curling deformation due to temperature variation of prestressed slab, is a distinctive phenomenon in slab track [9-11]. By building a computational model based on the temperature data of overhead track slab in Chongqing, Wang et al. [12] obtained the maximum warpage of the track slab $(0.82 \mathrm{~mm})$. Similarly, the maximum track slab warpage was calculated to be $0.327 \mathrm{~mm}$ according to the field test data from Beijing circular railway [13]. In some other research, the maximum warpage of track slab before laying rail was reported as $1.512 \mathrm{~mm}$ [14], $1.37 \mathrm{~mm}$ [15], $1.4 \mathrm{~mm}$ [16], etc. However, none of the above studies have taken into consideration the effects on the gap of rails and volume deformation of CA mortar layer and hence the maximum warpage values obtained cannot be applied in verifying finite element models for calculating the actual conditions of the slab warpage. In addition, previous studies $[17,18]$ have predicted that the gap will cause the slab to beat the filling layer repeatedly, thus leading to the damage of mortar and affecting the final track structure simultaneously.

In this work, in order to study the influence mechanism of rail on the gap, we selected CRTS I slab track in two different engineering conditions: track before laying rail and track after laying rail, as measuring objects. The following parameters were measured continuously over $24 \mathrm{~h}$ : (1) the temperature variation in different regions of the track slab by multi-channel wireless thermometers; (2) the elevation of track slab and the horizontal and vertical 
deformation of mortar by electronic level and dial gauge; and (3) the width and depth of the gap between track slab and mortar by feeler gauge and steel ruler, respectively. By investigating the gap at the four corners, we analyzed the initiation of gap and offered advices and guidance for structural design and construction of CRTS I slab track.

\section{Field test}

We selected two segments of the same experimental track constructed with the same CA mortar in order to keep identical filling layer conditions. After grouting the CA mortar and filling the resin around convex block over 28 days, we laid the rail on one segment but no rail on the other segment. The type of the slab is P4962 with a size of $4962 \mathrm{~mm} \times 2400 \mathrm{~mm} \times 200 \mathrm{~mm}$. The distribution of test sites on the track slab is shown in Fig. 1. Sites 1, 4, 5 and 8 were located near corners; sites 2, 3, 6 and 7 at the quarter points of two sides, while site 9 at the center. The field test was carried out in late August, 2010.

The elevation of slab was measured by electronic level (Leica DNA03) and dial gauge, the horizontal and vertical deformation of mortar were measured by dial gauge, the width of gap was measured by feeler gauge, and the depth of gap was measured by steel ruler.

HC-TW20 wireless thermometer, equipped with two transmitters and twelve data acquisition channels, was used to measure the temperature of mortar below the track slab. Before grouting CA mortar, we set sensors (1) at the top and bottom of grouting bag, (2) above the track slab, and (3) in the ambient environment, in order to collect data continuously after grouting.

\section{Results and analysis}

The temperatures of different test sites were measured at an offline technical experiment base for infrastructures. The elevation, gap and deformation of three track slab with rail

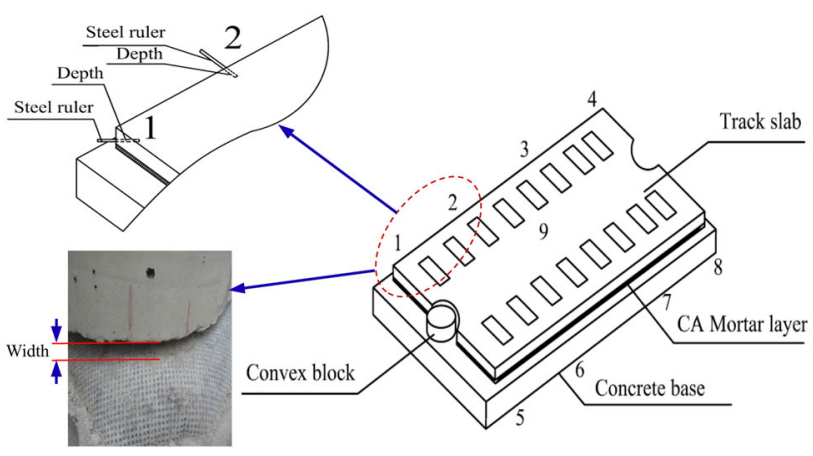

Fig. 1 Distribution of test sites laid (restrained by fasteners) and track slab without rail (free-state) were measured separately. Then, representative data were selected and analyzed.

\subsection{Temperature variation of different regions}

After the CA mortar was grouted, temperatures in different sites were tested over $24 \mathrm{~h}$ during the first 2 days, and solar radiation of the first day was much stronger than that of the second day. The temperatures collected automatically by the wireless thermometer are shown in Fig. 2.

Figure 2 shows that the track slab has the greatest temperature variation, followed by the ambient environment, while the mortar layer has the least variation. The temperature of track slab is higher than the ambient temperature but both of them share the same variation pattern and reach the lowest temperature during 04:00 to 07:00. The bottom surface of CA mortar has the highest temperature during 01:00 and 02:00, which is different from the ambient temperature. The possible reason for this difference is that the exothermic hardening process of CA mortar reaches the highest rate $10 \mathrm{~h}$ later after grouted, resulting in the highest temperature at midnight. The maximum temperature difference is $11.2{ }^{\circ} \mathrm{C}$ between the atmosphere and the bottom surface of slab (i.e., the top surface of CA mortar layer), while there is a difference of $8.3^{\circ} \mathrm{C}$ between the top surface and the bottom surface. The temperature at the top mortar surface is slightly higher than that at the bottom surface and the maximum difference is $2.6{ }^{\circ} \mathrm{C}$. The alteration of day and night caused a temperature gradient between the top and bottom surfaces of the slab, accordingly resulting in curling deformation of the slab.

\subsection{Elevation of track slab}

We put the electronic level on the top surfaces of track slabs with and without rail laid to measure the elevations at

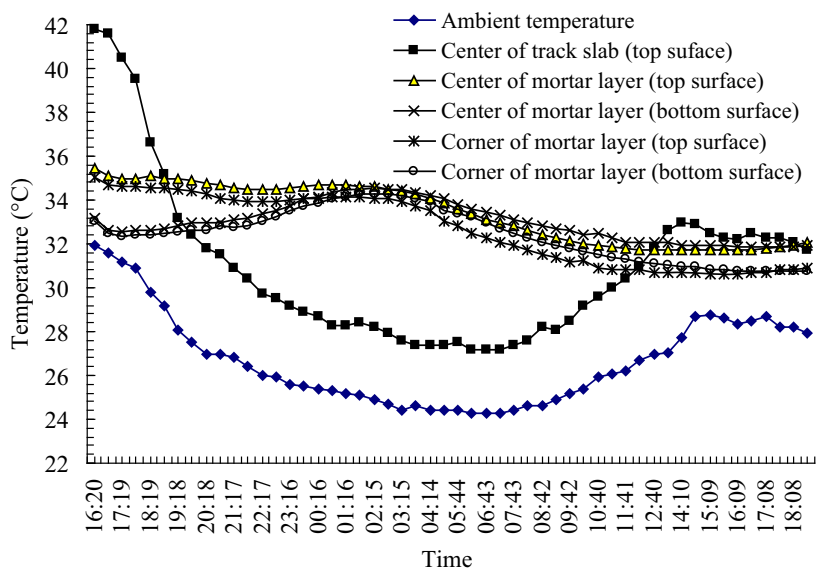

Fig. 2 Temperatures of environment, track slab and mortar 
the aforementioned nine test sites. Site 9 at the track center stands for the actual slab elevation, while the other eight sites are relative elevations compared to the value at site 9 . The measurements are presented in Figs. 3 and 4.

For the track slab without rail (Fig. 3), the elevation of the slab varies regularly with time. The track center has the lowest elevation during 04:00 and 06:00 and has the highest elevation at 12:00. Comparatively, the corners of track slabs curl at night and the maximum elevation difference between the center and the corner is $1.3 \mathrm{~mm}$. The quarter-point sites 2, 3, 6 and 7 also curl upward at night, but the curling deformation is $50 \%$ less than that of the corners.

The variation pattern of elevation at different test sites of the track slab with rail laid (Fig. 4) is generally similar to that of the track slab without rail. That is, the corners of track slab curl upward at night while the center concaves at the same time. Nevertheless, the extent of elevation variation of the track slab with rail declines considerably compared to that without rail. The maximum elevation of the slab center decreases from $0.3 \mathrm{~mm}$ in the case without rail to $0.07 \mathrm{~mm}$ in the case with rail. Meanwhile, the maximum relative elevation of corners decreases to $0.79 \mathrm{~mm}$, which is $40 \%$ less than that of the track slab without rail. In contrast, there is no significant decrease in curling extent in sites 2, 3, 6 and 7 at night, indicating that the rail could confine the deformation of track corners efficiently.

Combining Figs. 3 and 4 with Fig. 2, we can conclude that there is an alternating convex-concave curling deformation at the four corners and the track center. The reason for this phenomenon is that the poor thermal conductivity of mortar causes a temperature gradient in the vertical direction of track slab. When the temperature at the top surface is higher than that at the bottom surface, tensile stress occurs at the bottom surface and induces downward curling of the four corners; when the temperature at the top surface is lower than that at the bottom surface, there will be tensile stress at the top surface and four corners will curl upward.

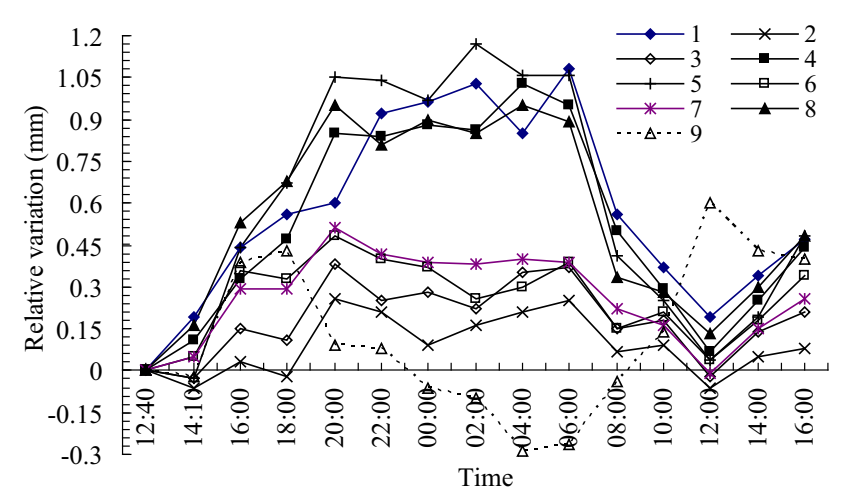

Fig. 3 Relative elevation variation of the track slab without rail laid

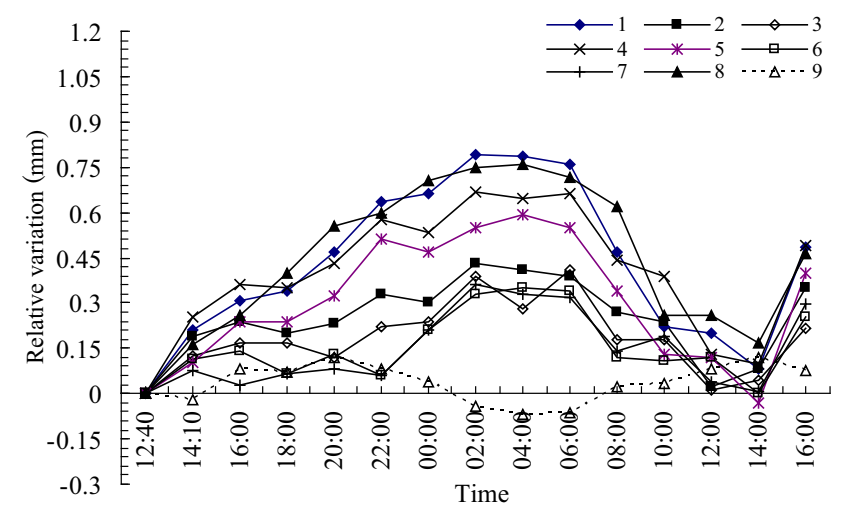

Fig. 4 Relative elevation variation of the track slab with rail laid

\subsection{Dial gauge measurement of corners}

Elevation variations at four corners of the track slabs with and without rail over $24 \mathrm{~h}$ were measured using dial gauge; the results are shown in Figs. 5 and 6.

As can be seen, the four corners of track slab curl upward significantly within $24 \mathrm{~h}$. Though the elevation values differ with corners slightly, their variation patterns are the same. The maximum values occur from 20:00 to $06: 40$. The variation pattern depicted by dial gauge measurements (Figs. 5, 6) is generally consistent with that by electronic level (Figs. 3, 4), but the specific value and time are slightly different. Comparing Figs. 5 with 6 , we find that the maximum elevation occurs at the corner of the track slab without rail is $1.61 \mathrm{~mm}$, whereas the maximum value of the track slab with rail laid is $0.86 \mathrm{~mm}$. The elevation difference of $0.75 \mathrm{~mm}(46 \%)$ indicates that rail can reduce the curl deformation of track slab effectively.

\subsection{Deformation of CA mortar}

Theoretically, the deformation of CA mortar will affect the gap. Figure 7 shows the gap depths at four corners of the

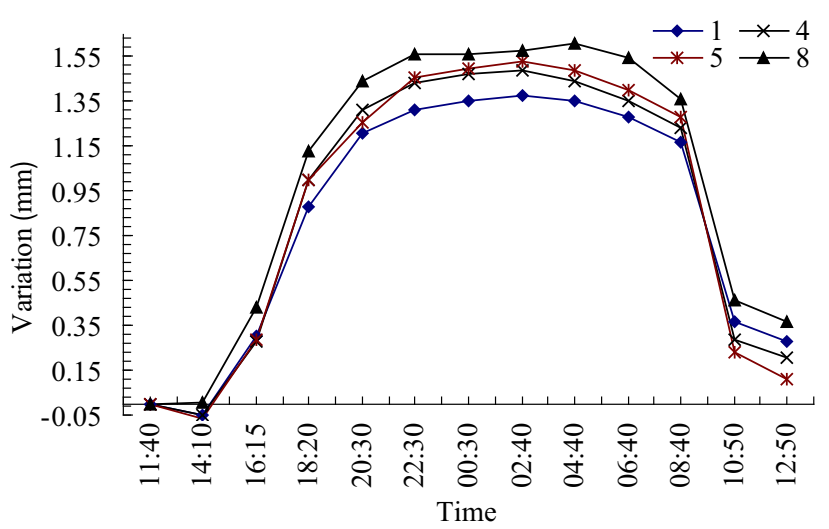

Fig. 5 Elevation variations of the track slab with rail laid 


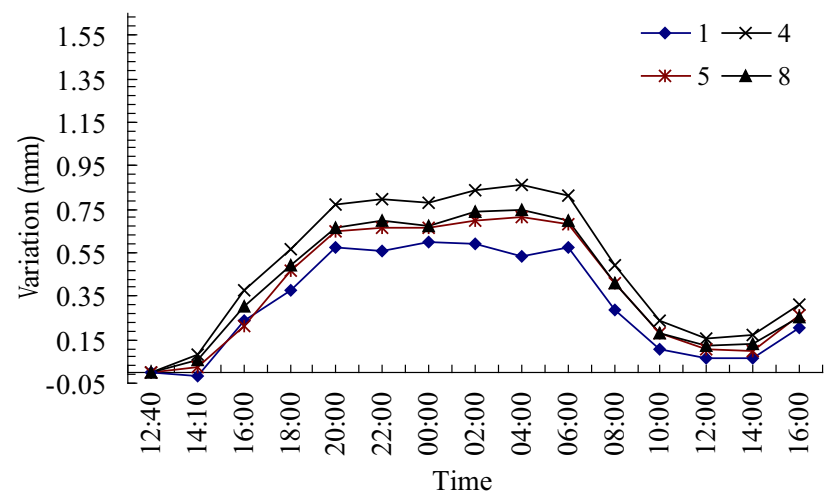

Fig. 6 Elevation variations of the track slab without rail

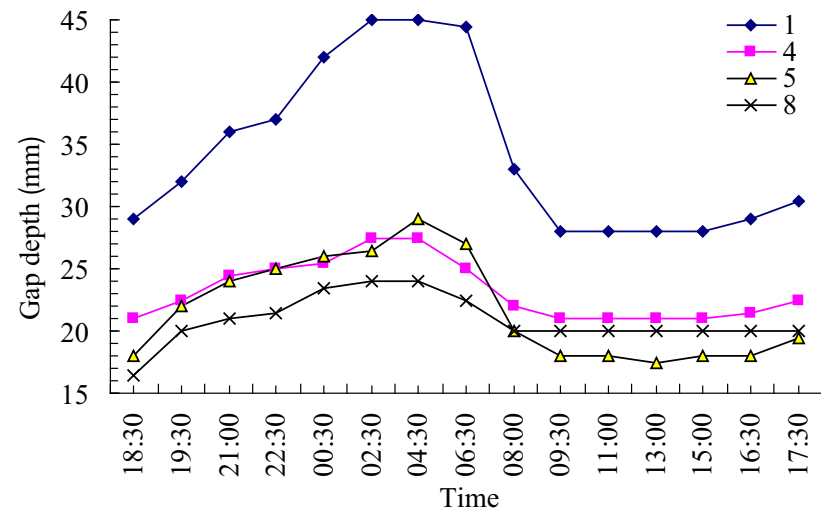

Fig. 7 Depth of gaps at four corners of the track slab without rail

track slab without rail, while Fig. 8 presents the horizontal and vertical deformation of mortar at the four corners, measured by dial gauge, on the same track slab.

We can see that from Fig. 7 that values of the gap depths at four corners are different, and the gap depth increases as the temperature decreases at night. Site 1 with the maximal initial gap depth of $45 \mathrm{~mm}$ has the maximum increase of $16 \mathrm{~mm}$ from $18: 30$ to $02: 30$. As the temperature increases

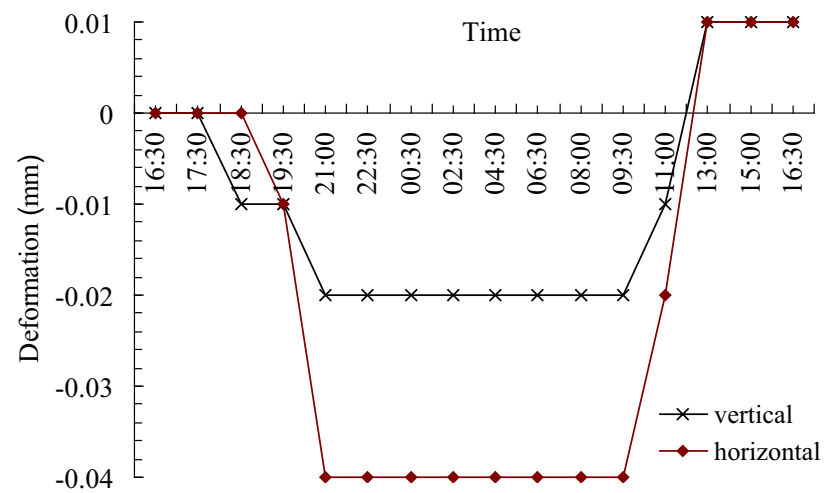

Fig. 8 Deformation of mortar at four corners of track slab without rail in the next early morning, the gap depth turns to decrease and becomes stable from 09:30 to 15:00; afterwards the gap depth increases again.

CA mortar layer has the maximum shrinkage from 21:00 to $09: 30$ (Fig. 8). The duration of shrinkage is longer than that of the maximum gap depth. Besides, when the temperature goes down to the lowest point, both the maximum horizontal shrinkage $(0.04 \mathrm{~mm})$ and the maximum vertical shrinkage $(0.02 \mathrm{~mm})$ occur, which is merely $2.7 \%$ of the curling elevation of track slab. Therefore, the slab curling caused by temperature gradient is independent from the temperature of CA mortar.

\subsection{Width and depth of gap}

The width and depth of gap at test sites 1-8 on track slab were measured using feeler gauge and steel ruler, respectively, on another track segment to explore the influence of rail laid. The results for track slabs with and without rail are presented in Figs. 9, 10, 11 and 12.

As shown in Figs. 9 and 10, the variation patterns of gap width in the two different conditions are similar. The width of gap at the corner increases considerably at night while the quarter points at the two track sides show a negligible increase. Without rail laid on the track slab, the maximum width of gap at corners is $1.75 \mathrm{~mm}$, which occurs from $22: 30$ to $06: 40$. After rail is laid on the track slab; the maximum width is $1.3 \mathrm{~mm}$, about $25.7 \%$ less than that of the track slab without rail in the same period.

When the depth of gap is evaluated by inserting a steel ruler, the depth varies obviously at corners but the variation can be neglected at the quarter points (Figs. 11, 12). Without the rail (Fig. 11), the gap at site 1 maintains at the greatest value about $49 \mathrm{~mm}$ from 02:00 to 06:00, which meets the acceptance standard (less than $50 \mathrm{~mm}$ ). Consistent with Fig. 7, the maximum value of depth is $16 \mathrm{~mm}$ larger than the initial value, while the depth at the quarter points remains almost unchanged. For track slab with rail

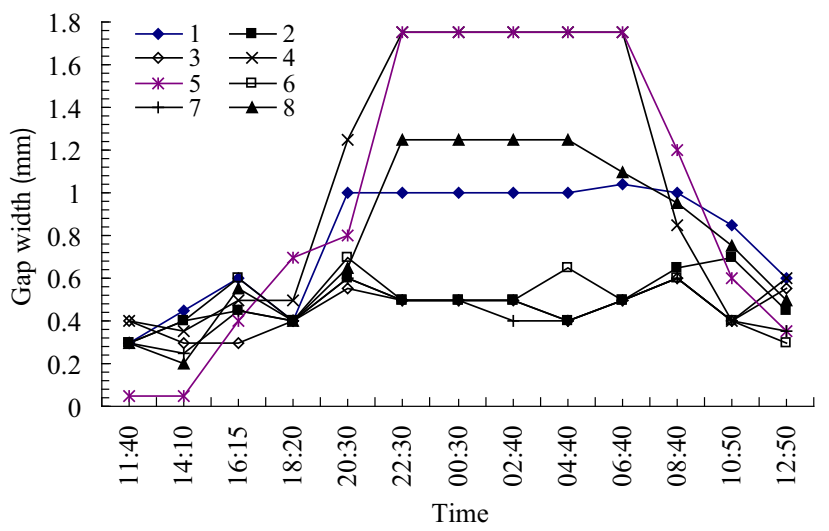

Fig. 9 Gap width of track slab without rail 


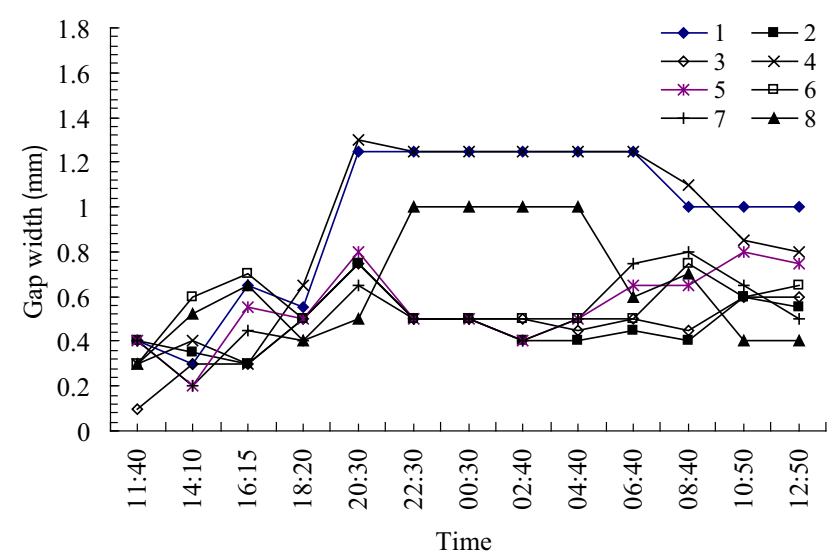

Fig. 10 Gap width of track slab with rail laid

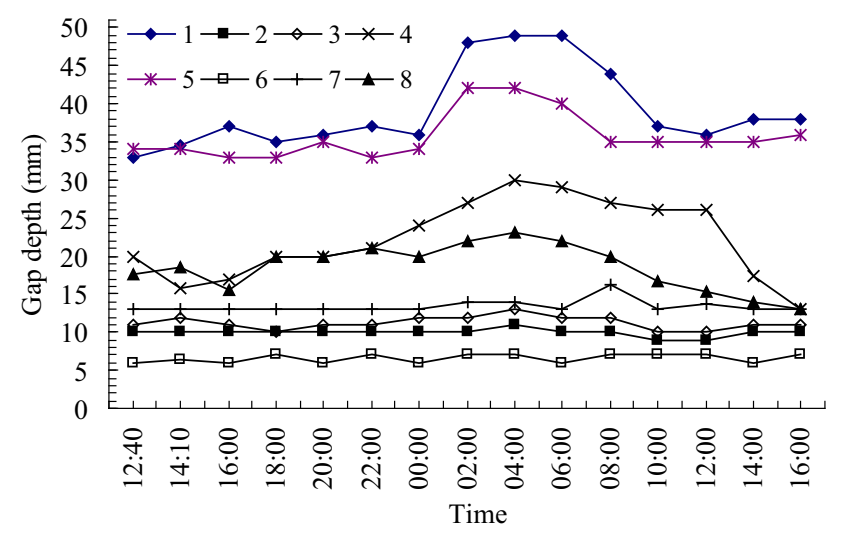

Fig. 11 Gap depth of track slab without rail

laid (Fig. 12), the maximum depth is $19.5 \mathrm{~mm}$ at 04:00, while the initial depth is $13 \mathrm{~mm}$. The difference between the initial and the maximum values is $6.5 \mathrm{~mm}$, which is $9.5 \mathrm{~mm}(59.4 \%)$ less than that of track slab without rail. The test results of gap width and depth indicate that rail can efficiently diminish the gap.

\subsection{Site investigations of existing lines}

We investigated the gap of 101 pairs of track slabs with rail, filled with CA mortar (404 test sites altogether), along the Harbin-Dalian passenger dedicated line, using $50 \mathrm{~mm}$ as the acceptance standard for gap depth of steel ruler. Results show that there were 9 slabs with 24 corners (5.9\%) exceeding this standard, and the maximum depth among them was $260 \mathrm{~mm}$. As shown in Fig. 13, the qualified corners accounted for $94.1 \%$ and most of the gap depths were less than $30 \mathrm{~mm}$.

At the same time, before laying the rail, Harbin-Dalian Passenger Special Line Company did a special inspection on the gap and investigated 20,226 test points, in which 2337 points $(11.6 \%)$ exceeded the standard of $50 \mathrm{~mm}$ for

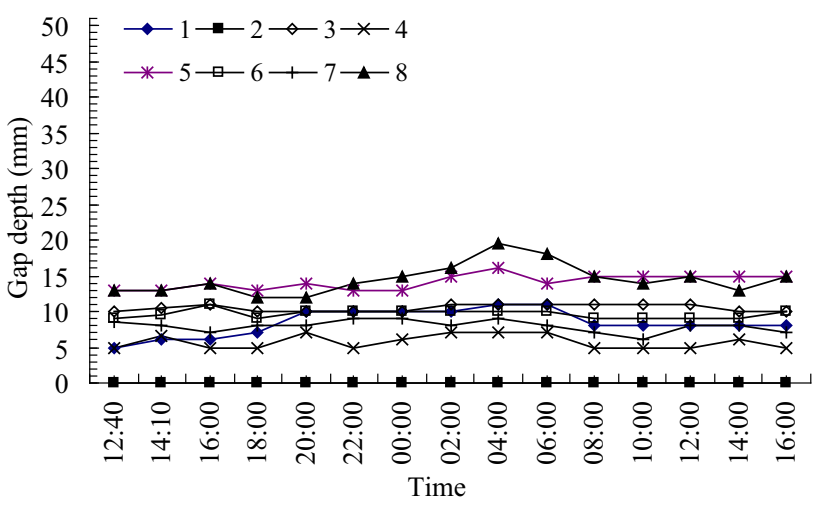

Fig. 12 Gap depth of track slab with rail laid

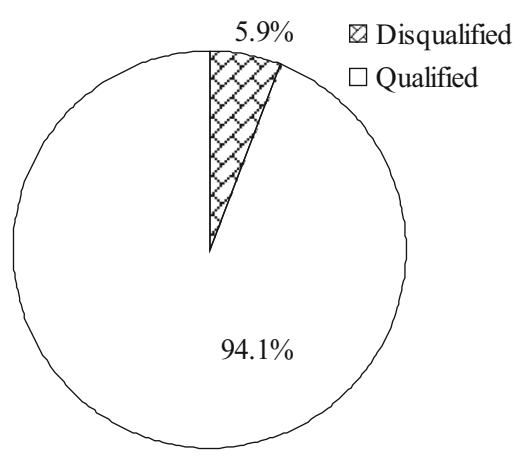

Fig. 13 Gap depth statistics of track slabs with rail laid

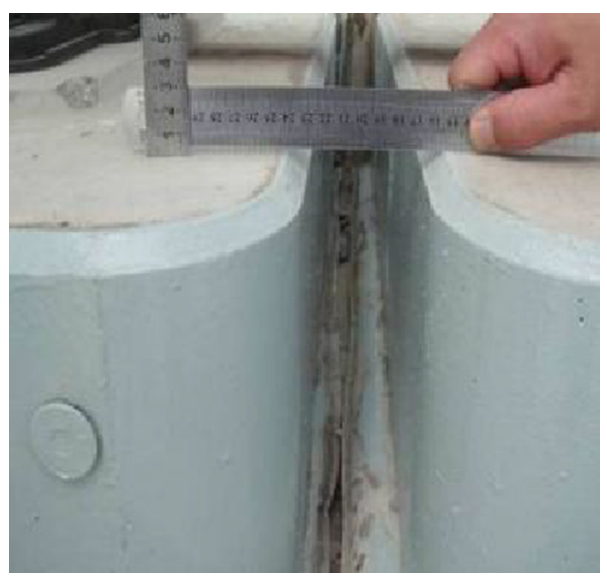

Fig. 14 The $4 \mathrm{~mm}$ mismatch between two slabs

acceptance [19]. After laying rail, the disqualification ratios dropped by $50 \%$, demonstrating that laying rail can effectively diminish the probability of unacceptable gap.

From the site investigation, we found that a corner with a large gap often had an elevation mismatch with its neighboring slab, as shown in Fig. 14. After removing the fasteners, the slab resumed partly, and there would be a width of $2 \mathrm{~mm}$ between the rail and the fastener. This kind of mismatch in elevation had also been described in [16]. 
Due to the mismatch, the lower slab will be dragged up by the higher slab, leading to the initiation of gap after laying the rail. Therefore, fine adjustment, fully filled CA mortar in filling space, and prevention of track slab floating are critical steps in constructing CRTS I slab track. When laying the rail, in order to avoid the lower slab being dragged up by the higher slab and the further occurrence of new gap, it is suggested to measure the elevation twice and adjust the lower slab with gasket according to the operation guide.

\section{Conclusion}

1. There exists a temperature gradient between the top surface and bottom surface of the track slab, resulting in upward curling at night and initiation of the new gap. The gap at four corners is more severe than that at other track parts distinctly.

2. The rail can efficiently decrease the size of gap. Compared with the track slab without rail, the maximum elevation at the corners of track slab with rail measured by dial gauge decreases by $0.75 \mathrm{~mm}$ $(46.6 \%)$, the maximum width measured by feeler gauge decreases by $0.45 \mathrm{~mm}(25.7 \%)$, and the maximum depth measured by steel ruler decreases by $9.5 \mathrm{~mm}(59.4 \%)$.

3. Against the $50 \mathrm{~cm}$ standard for gap depth, for the Harbin-Dalian passenger dedicated line, the disqualification ratio of track slabs after laying rail is 5.9\%, which is $50 \%$ lower than that of the track before laying rail. When the elevation mismatch occurs, a gasket can be placed at rail-bearing bed of the lower track slab in order to avoid the lower slab being dragged up by the higher slab and the resultant occurrence of new gap.

4. Fine adjustment, fully filled CA mortar in fillings pace and prevention of track slab floating are critical steps in constructing CRTS I slab track. When laying rail, it is suggested to measure the elevation twice and adjust the lower slab with the gasket according to the operation guide.

Acknowledgements This work was supported by the National Natural Science foundation of China (No. 51408610).

Open Access This article is distributed under the terms of the Creative Commons Attribution 4.0 International License (http:// creativecommons.org/licenses/by/4.0/), which permits unrestricted use, distribution, and reproduction in any medium, provided you give appropriate credit to the original author(s) and the source, provide a link to the Creative Commons license, and indicate if changes were made.

\section{References}

1. Shigeru M, Hideyuki T, Masao U et al (1998) The mechanism of railway tracks. Jpn Railw Transp Rev 3:38-45

2. Katsuoshi A (2001) Development of slab tracks for Hokuriku Shinkansen line. Q Rep RITI 42(1):35-41

3. Gautier P (2015) Slab track: review of existing systems and optimization potentials including very high speed. Constr Build Mater 92:9-15

4. Murata O (2003) Overview of recent structure technology R\&D at RTRI. Q Rep RITI 44(4):133-135

5. Li PG, Liu XY, Li GQ (2014) Influence of CA mortar void on dynamic characteristics of unit slab track on bridge. China Railw Sci 35(3):20-27 (in Chinese)

6. Ren J, Li X, Yang R et al (2016) Criteria for repairing damages of CA mortar for prefabricated framework-type slab track. Constr Build Mater 110:300-311

7. Zeng XH, Xie YJ, Deng DH (2012) Effect of Temperature on early expanding character of cement and emulsified asphalt mortar. J Chin Ceram Soc 40(2):207-211 (in Chinese)

8. Liu Y, Zhao GT (2013) Analysis of early gap between layers of CRTS II slab ballastless track structure. China Railw Sci 33(4):1-6 (in Chinese)

9. Wang FZ, Liu ZC, Hu SG (2010) Early age volume change of cement asphalt mortar in the presence of aluminum powder. Mater Struct 43:493-498

10. Wang T, Jia HQ, Li HG et al (2016) Research on water seepage of cement asphalt emulsified (CA) mortar. Constr Build Mater 125:595-599

11. Zhu S, Cai C (2014) Interface damage and its effect on vibrations of slab track under temperature and vehicle dynamic loads. Int $\mathrm{J}$ Non-Linear Mech 58:222-232

12. Wang SR, Sun L, Li QY et al (2009) Temperature measurement and temperature stress analysis of Ballastless Track Slab. J Railw Eng Soc 2:52-55 (in Chinese)

13. Wang JJ, You RL, Wang M et al (2010) Research on the slab temperature warping of the unit slab track system. China Railw Sci 31(3):9-14 (in Chinese)

14. Shao PY, Li HY, Wu SL et al (2013) Measurement and research on temperature warping of CRTS I track slab and crack between track slab and cement asphalt mortar cushion. China Railw Sci 34(2):18-22 (in Chinese)

15. Yi T, Zhao Q, Xiao W (2012) Analysis of temperature deformation and stress value of CRTS I track slab on Harbin-Dalian Passenger dedicated line. Railw Stand Des 5:37-39 (in Chinese)

16. Lu W (2014) Research on the seams genesis of CRTS-I Ballastless Track Slab's four corners in severe cold area. J Railw Eng Soc 31(7):53-56 (in Chinese)

17. Wang $\mathrm{P}, \mathrm{Xu} \mathrm{H}$, Chen $\mathrm{R}$ (2016) Effect of cement asphalt mortar debonding on dynamic properties of CRTS II slab ballastless track. Adv Mater Sci Eng 2014(2):1-8

18. Yuan Q, Liu W, Pan Y et al (2016) Characterization of cement asphalt mortar for slab track by dynamic mechanical thermoanalysis. J Mater Civ Eng 28(3):04015154

19. The Company of Harbin-Dalian Passenger Dedicated Line (2010) Investigation report on gap between layers of CRTS-I Ballastless Track. The Company of Harbin-Dalian Passenger Dedicated Line, Shenyang (in Chinese) 